\title{
Gain-of-function mutation in Gnao1: A murine model of epileptiform encephalopathy (EIEE17)?
}

\author{
Jason M. Kehrl · Kinshuk Sahaya • Hans M. Dalton • Raelene A. Charbeneau • \\ Kevin T. Kohut • Kristen Gilbert • Madeline C. Pelz • Jack Parent • \\ Richard R. Neubig
}

Received: 15 February 2014/Accepted: 11 March 2014/Published online: 5 April 2014

(C) Springer Science+Business Media New York 2014

\begin{abstract}
G$ protein-coupled receptors strongly modulate neuronal excitability but there has been little evidence for $\mathrm{G}$ protein mechanisms in genetic epilepsies. Recently, four patients with epileptic encephalopathy (EIEE17) were found to have mutations in GNAO1, the most abundant $\mathrm{G}$ protein in brain, but the mechanism of this effect is not known. The GNAO1 gene product, $\mathrm{G} \alpha_{\mathrm{o}}$, negatively regulates neurotransmitter release. Here, we report a dominant murine model of Gnaol-related seizures and sudden death. We introduced a genomic gain-of-function knock-in mutation $\left(\right.$ Gnaol $\left.^{+/ \mathrm{G} 184 \mathrm{~S}}\right)$ that prevents $\mathrm{G}_{\mathrm{o}}$ turnoff by Regulators of $\mathrm{G}$ protein signaling proteins. This results in rare seizures, strain-dependent death between 15 and 40 weeks of age, and a markedly increased frequency of interictal epileptiform discharges. Mutants on a C57BL/6J background also have faster sensitization to pentylenetetrazol
\end{abstract}

Electronic supplementary material The online version of this article (doi:10.1007/s00335-014-9509-z) contains supplementary material, which is available to authorized users.

J. M. Kehrl · H. M. Dalton · R. A. Charbeneau ·

K. T. Kohut - K. Gilbert - M. C. Pelz

Department of Pharmacology, University of Michigan,

Ann Arbor, MI 48109, USA

K. Sahaya $\cdot$ J. Parent

Department of Neurology, University of Michigan, Ann Arbor, MI 48109, USA

J. Parent

Ann Arbor Veterans Administration Healthcare System,

Ann Arbor, MI 48105, USA

R. R. Neubig $(\square)$

Department of Pharmacology \& Toxicology, Michigan State University, B440 Life Sciences, 1355 Bogue St, East Lansing, MI 48824, USA

e-mail: rneubig@msu.edu
(PTZ) kindling. Both premature lethality and PTZ kindling effects are suppressed in the $129 \mathrm{SvJ}$ mouse strain. We have mapped a 129S-derived modifier locus on Chromosome 17 (within the region 41-70 MB) as a Modifer of $\mathrm{G}$ protein Seizures (Mogs1). Our mouse model suggests a novel gainof-function mechanism for the newly defined subset of epileptic encephalopathy (EIEE17). Furthermore, it reveals a new epilepsy susceptibility modifier Mogs 1 with implications for the complex genetics of human epilepsy as well as sudden death in epilepsy.

\section{Introduction}

Epilepsy is a common but complex neurological disorder affecting approximately $0.7 \%$ of the population (Hirtz et al. 2007), but nearly one-third lack effective treatment (Engel 1996; Loscher 2011). Multiple genetic factors cause or contribute to the severity of epilepsy (Michelucci et al. 2012), so understanding mechanisms that can modify seizure occurrence or severity could reveal novel epilepsy drug targets. While many mutations that cause human epilepsy are in ion channels, the recent description of de novo mutations in GNAO1 (Nakamura et al. 2013) in early infantile epileptiform encephalopathy (EIEE17, MIM 615473 ) is intriguing since $\mathrm{G}$ protein-coupled receptors are valuable drug targets.

It has been known for some time that G-protein-coupled receptor (GPCR) signaling plays an important role in epilepsy. These receptors activate heterotrimeric G-proteins which consist of a $\mathrm{G} \alpha$ subunit and a $\mathrm{G} \beta \gamma$ dimer. Gnaol encodes for the alpha subunit of $G_{o}, a G_{i / o}$ family member $\left(\mathrm{G}_{\mathrm{i} 1}, \mathrm{G}_{\mathrm{i} 2}, \mathrm{G}_{\mathrm{i} 3}, \mathrm{G}_{\mathrm{o}}\right)$ that is highly abundant in brain (Birnbaumer 2007). This family of proteins inhibits adenylyl 
cyclase and can reduce neuronal excitability through regulation of ion channels. Blockade of the entire $\mathrm{G}_{\mathrm{i} / \mathrm{o}}$ family with pertussis toxin enhances seizure susceptibility (Ormandy and Jope 1991). $G_{o}$, in particular, plays a key role in presynaptic inhibition of neurotransmitter release through a $\mathrm{G} \beta \gamma$-mediated suppression of voltage-gated $\mathrm{Ca}^{2+}$ channels (Chen and Lambert 2000). Also, $G_{0}$ is activated by the A1 adenosine receptor ( $\mathrm{Fu}$ et al. 2006) which is protective in several seizure models (Chen et al. 2012; Li et al. 2007; Masino et al. 2011). This suggests that enhancing $G \alpha_{o}$ function might reduce seizure activity, consistent with our previous study showing reduced repetitive firing in a hippocampal slice model (Goldenstein et al. 2009).

However, this simplified in vitro system seems not to reflect the complex nature of epilepsy in vivo. Indeed acute seizure models (Loscher 2011) do not effectively assess epileptogenesis, the maladaptive changes that occur after initial excitatory stimuli which lead to further enhancement of seizure susceptibility. Epileptogenesis is thought to play a key role in the progression of human epilepsy (Morimoto et al. 2004). Electrical or chemical kindling models (Bialer and White 2010) attempt to assess this with repeated subthreshold excitatory stimuli that eventually result in generalized seizures. Pentylenetetrazol (PTZ) is a non-competitive $\mathrm{GABA}_{\mathrm{A}}$ antagonist. A single large dose induces seizures by engaging the brainstem (Peterson and Albertson 1998), however, lower doses of PTZ, in a repeateddose kindling protocol, preferentially activate the deep prepiriform cortex (Peterson and Albertson 1998). This causes remodeling that parallels change in human epileptic brains such as mossy fiber sprouting within the dentate gyrus (Golarai et al. 1992; Lee et al. 2012).

Here, we describe a mouse model with features that are shared with the recently described EIEE17 syndrome of human epilepsy (Nakamura et al. 2013). This heterozygous Gnaol $^{+/ \mathrm{G} 184 \mathrm{~S}}$ mutation produces early neonatal and adult lethality accompanied by epileptiform discharges, markedly enhanced sensitivity to PTZ-induced kindling, and rare spontaneous seizures. Furthermore, we have identified a Chr17 modifier that affects both PTZ kindling and spontaneous death in our model. This animal model, as well as the modifer locus on $\mathrm{Chr} 17$, should provide insights into mechanisms of a human epileptiform encephalopathy as well as potential therapeutic approaches.

\section{Materials and methods}

Animal studies were performed in accordance with the Guide for the Care and Use of Laboratory Animals established by the National Institutes of Health, and all experimental protocols were approved by the University of Michigan Committee on the Use and Care of Animals.
Detailed experimental protocols are outlined in the Supplemental Information but key information is presented here.

Generation of Gnao1 G184S mutant mice

The generation of the Gnaol G184S knock-in mice (tm2Rneu) allele from a CJ7 bac library into the 129-steel derived ES line, CJ7, was previously reported (Goldenstein et al. 2009) with additional details in Supplemental Information. Breeding on all genetic backgrounds was performed with Gnaol ${ }^{+/ t m 2 R n e u}$ male and Gnaol $^{+/+}$females. As described in results, homozygotes were non-viable so all studies were done with heterozygous mutants. To obtain mutant mice on the B6 background, the tm2Rneu allele, after Neo excision, was successively backcrossed onto the C57BL/6J background (Jackson Labs stock \# 000,664). A single N6 B6-Gnaol $1^{+/ \text {tm2Rneu }}$ male ancestor gave rise to all N7-N9 B6-Gnao1 ${ }^{+/ t m 2 R n e u}$ mice presented in this study which will be termed B6-Gnaol ${ }^{+/ G 184 S}$. Due to reduced viability and premature lethality of the B6-Gnaol ${ }^{+/ \mathrm{G} 184 \mathrm{~S}}$ mutants, we were not able to generate sufficient $\mathrm{N} 10+$ mice for studies. F1 mice (B6-129S-F1) carrying the Gnaol $^{+/ \mathrm{G} 184 \mathrm{~S}}$ allele were generated from crosses of Gnaol $^{+/ G 184 S}$ 129S1/SvImJ male mice with C57B1/6J females. Female mice from F2 crosses (F1 brother-sister matings) were also studied for survival.

\section{Genotyping}

The G184S allele of Gnaol was identified by genomic PCR as described (Goldenstein et al. 2009) with details in Supplemental Information.

\section{Viability}

Adult survival studies were done with group-housed B6, 129, F1, or F2 Gnaol $^{+/ G 184 S}$ male or female mice as indicated. Female B6 Gnaol ${ }^{+\mathrm{G} 184 \mathrm{~S}}$ mutant mice also underwent video recording in their cages around the clock for up to 10 weeks using an IR camera. Cages were inspected each morning and tapes were reviewed in the event of a death within the cage.

\section{Video EEG}

Female mutant and control mice approximately 24 weeks old were implanted with electrodes, given one week to recover, and monitored under video EEG as described by Lee et al. (2012) with additional details in Supplemental Information. Interictal epileptiform discharges (IEDs) were assessed by manual analysis by a blinded observer since automated spike detection gave a high proportion of false 
positive and false negative calls. IEDs were defined as waveforms lasting 20-200 ms, at least 3 times the background activity in amplitude, disturbing the background.

\section{Chemical kindling susceptibility}

A PTZ kindling protocol was performed essentially as described (Wilczynski et al. 2008) to assess epileptogenesis. Briefly, PTZ was administered 3 times per week at $35 \mathrm{mg} / \mathrm{kg}$ starting at 8-12 weeks of age; mice were monitored for $30 \mathrm{~min}$ for behavioral signs of seizures as described (Dhir 2012; Grecksch et al. 2004; Wilczynski et al. 2008). This dose seldom elicits seizures on the first dose in wild-type mice regardless of gender or strain (lab observation). Sensitization is defined as death or two sequential sessions with a tonic-clonic seizure. The number of injections for each mouse to reach a sensitized state is reported in survival curves.

\section{Statistical analysis}

Data were analyzed using GraphPad Prism 5.0 and GraphPad QuickCalcs (GraphPad; LaJolla, CA). Proportionality of hazards for survival curves was analyzed using SAS 9.3 (SAS Institute; Cary, NC). Chi squared analysis was used to evaluate deviation from Mendelian ratios. The Gehan-Breslow-Wilcoxon test was applied to survival and kindling data since survival curves failed to meet the proportional hazard assumption required for logrank tests (Machin et al. 2006). For EEG data, a parametric, an unpaired $t$ test was performed with Welch's correction for unequal standard deviations. Two tailed $p$ values less than 0.05 were considered significant.

\section{Results}

Gnaol G184S mutant mice die perinatally

In light of the de novo mutants of GNAO1 recently reported in early infantile epileptiform encephaolopathy (Nakamura et al. 2013), we reexamined previously described mice carrying the G184S Gnaol mutant gain-offunction allele (tm2Rneu-MGI:5508226) (Goldenstein et al. 2009). Heterozygous mutant mice are viable but obtained in reduced frequencies, while homozygotes are essentially not viable. From het $\times$ het crosses of Gnaol G184S mutants on the 129 strain background, we obtained at weaning: $20 \mathrm{wt}, 19$ het, and only 2 homozygous mutants (5\%, 1 male and 1 female). Both homozygous and heterozygous mutants were underrepresented (Fig. S1A Chi squared $\left.\chi^{2} \operatorname{df}(2)=16.02 p<0.001\right)$. Similarly, after backcrossing four times onto a C57BL/6J background (N4 B6), no homozygotes survived to weaning out of fifty-five offspring from het $\mathrm{x}$ het crosses and hets were substantially underrepresented ( 21 het vs $34 \mathrm{wt}$; expected value for hets is $2 \times$ WT or 68; Fig. S1B Chi squared $\chi^{2} \mathrm{df}(2)=45$, $p<0.0001)$. Sex did not influence genotype distributions on either background (data not shown).

To understand the loss of viability of mice carrying the Gnaol G184S allele, timed pregnancies were performed for N7 B6 het $\mathrm{x}$ het crosses and embryos were collected. At E18.5, genotypes did not differ from the expected Mendelian ratio (Fig. S1C), indicating that the loss of viability occurred after E18.5 but before 2 weeks of age. With close monitoring of litters, there were very few viable homozygous offspring and hets were also underrepresented (Fig. S1D, Chi squared $\left.\chi^{2} \operatorname{df}(2)=8.76 p<0.02\right)$. Many homozygotes and some hets were found dead but there were no obvious anatomical abnormalities. Nissl staining performed on brains of E18.5 embryos revealed no major defects in brain structure (Fig. S2A-d). G $\alpha_{o}$ protein expression in the brains of hets at 8-12 weeks of age was found to be normal, indicating that the mutation was not significantly disrupting protein levels (Fig. S2e-f).

Heterozygous Gnaol ${ }^{+/ \mathrm{G} 184 \mathrm{~S}}$ mice experience sudden death

Gnaol G184S B6 hets that survived to weaning ( 30\% of expected) experience lethality starting at $\sim 80$ days (11-12 weeks) with $\sim 50 \%$ dead by 25 weeks of age (Fig. 1a). This was due to the Gnaol mutant allele rather than to strain background as wild-type littermates survived to greater than 1 year (data not shown). Interestingly, there appeared to be heterogeneity in survival as the $\sim 50 \%$ that survived to 25 weeks showed little further premature lethality.

To understand the cause of death, mice were monitored three times weekly in daylight hours over 2 months. There were no evident signs of illness, weight loss, distress, or seizures prior to death. However, using continuous IR video recording of B6 female Gnaol G184S mutant mice over $\sim 900$ mouse-days, we captured two deaths. Females were chosen for these studies due to the ability to group hour up to four mice for these long-term studies. In one instance, there was an apparent seizure with rearing and wild jumping (Fig. 1c and video supplement) observed during the night prior to the mouse being found dead in the cage. The second death showed no evident seizure.

Heterozygous Gnaol ${ }^{+/ \mathrm{G} 184 \mathrm{~S}}$ mice have EEG abnormalities

We also assessed video EEGs of B6 females (Fig. 1d, e). Mutant mice (age $\sim 24$ weeks) had a substantially elevated frequency of interictal epileptiform discharges (IEDs; 363, 

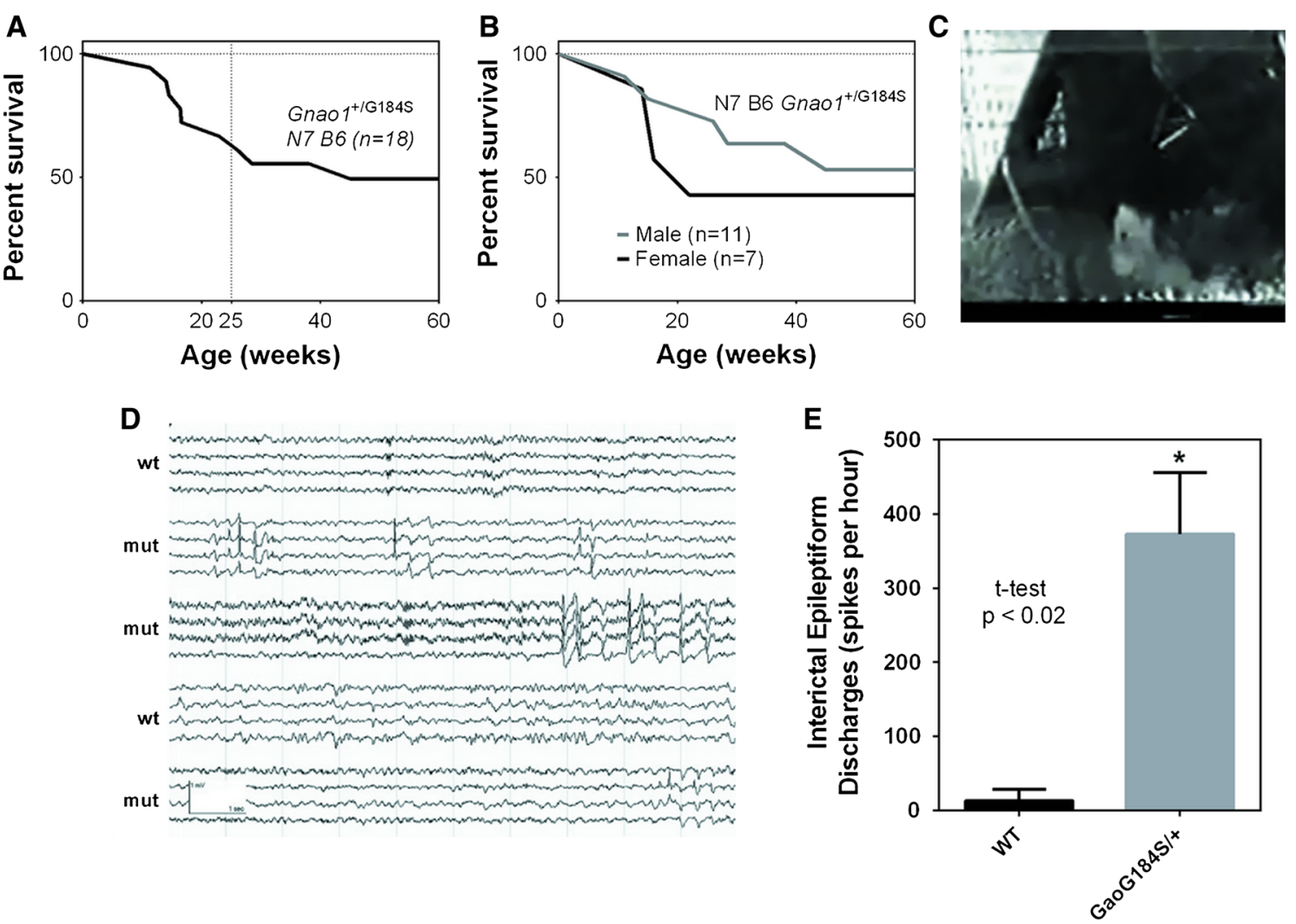

Fig. 1 Gnaol $^{+/ G 184 S}$ mice exhibit strain-dependent lethality, seizures, and altered EEGs. a Gnaol ${ }^{+/ G 184 S}$ mutant mice on a C57BL/6J background (N7 B6) die prematurely (Gehan-Breslow-Wilcoxon $\operatorname{df}(1)=12.8, p<0.001)$. b Early lethality is independent of gender. c Video monitoring revealed a seizure just prior to death (video in supplement). d, e EEG monitoring of Gnaol mutant and wt mice

296 and 460 IEDs/h for hets versus 2 and 24 IEDs/h for controls-unpaired $t$ test $t=7.37 \mathrm{df}=2.2, p<0.02$ ). The IEDs seemed to occur more frequently during electrographic sleep than during wakefulness. Frequently, they occurred in runs of $1-3 \mathrm{~Hz}$, but over the 4-8 day monitoring period, none met criteria for frank electrographic seizures due to the short duration the discharges $(<10 \mathrm{~s})$ nor were any overt seizures seen on video over this time period.

Heterozygous Gnaol $^{+/ \mathrm{G} 184 \mathrm{~S}}$ mice are sensitized to PTZ kindling

To provide an independent and earlier assessment of the susceptibility to epileptogenesis in the mutant mice, we performed a PTZ kindling model in 8-12 week old mice (see "Materials and Methods"). Female B6 Gnaol ${ }^{+/ \mathrm{G} 184 \mathrm{~S}}$ mutants were more sensitive to PTZ kindling than were controls (Fig. 2a Gehan-Breslow-Wilcoxon $\mathrm{df}(1)=5.74$, $p<0.02$ ). This was independent of gender (similar to demonstrate a greater than tenfold increase in interictal epileptiform discharges (IEDs) in B6 Gnaol $^{+/ \mathrm{G} 184 \mathrm{~S}}$ mutant females compared to littermate wt controls (unpaired $t$ test $t=7.37 \mathrm{df}=2.2, p<0.02$ ). Vertical bars demark the passage of $1 \mathrm{~s}$. Data represent mean \pm SEM. $* p<0.02$

spontaneous death) as mutant males also showed enhanced PTZ sensitization (Fig. 2b, Gehan-Breslow-Wilcoxon $\mathrm{df}(1)=6.88, p<0.01)$.

To determine whether the cause of seizure susceptibility in our mice might be due to loss of function of Gnaol as suggested for humans in EIEE17 (Nakamura et al. 2013), we evaluated Gnaol knockout mice. The homozygotes show early lethality (Valenzuela et al. 1997) and we were unable to generate sufficient numbers to undertake studies. The heterozygous knockout $\left(\mathrm{Gnaol}^{+/-}\right)$mice showed no enhancement in sensitivity to PTZ kindling (Fig. 2c). The wild-type mice did show earlier seizure onset than the WT controls for the Gnaol G184S mutants. This was unexpected. The most plausible explanation is that the two lines were developed from separate colonies and the backgrounds may be somewhat different despite both being C57Bl/6. The lack of increased kindling in the Gnaol $^{+/-}$mice combined with the normal level of expression of $\mathrm{G} \alpha_{\mathrm{o}}$ in our Gnaol G184S mutants suggests that loss of function in our mutants does not explain our results (see "Discussion"). 

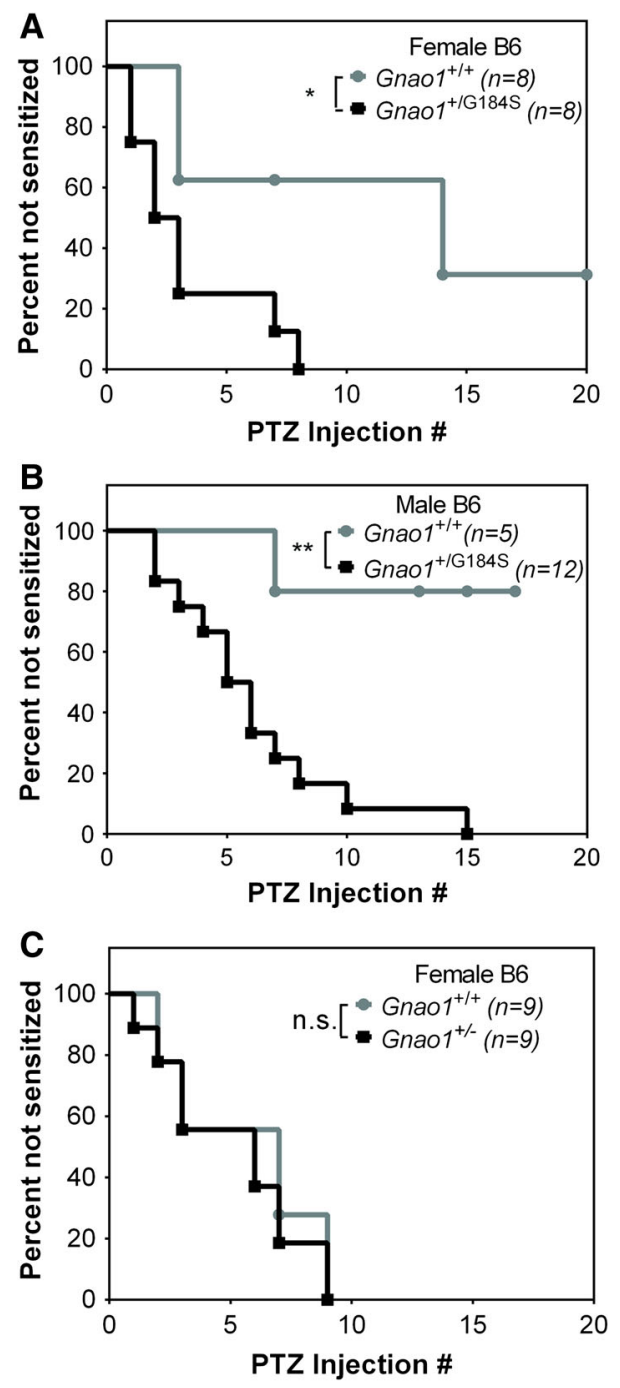

Fig. 2 Gnaol $^{+/ G 184 S}$ mice sensitize faster to repeated low-dose PTZ. The time to undergo sensitization to PTZ kindling (see Methods for definition) was recorded. a B6 female Gnaol $^{+/ \mathrm{G} 184 \mathrm{~S}}$ sensitize more rapidly than littermate controls (Gehan-Breslow-Wilcoxon $\operatorname{df}(1)=5.74, p<0.05)$. b Similarly Gnao $^{+/ \mathrm{G} 184 \mathrm{~S}}$ males also sensitize more rapidly than littermate controls (Gehan-Breslow-Wilcoxon $\operatorname{df}(1)=6.88, p<0.01)$. c B6 heterozygous knockout Gnaol ${ }^{+/-}$ mutant females do not sensitize more rapidly than littermate controls $(p>0.05)$

Both spontaneous death and increased sensitivity to epileptogenesis are strain dependent

Interestingly, Gnaol G184S heterozygous mutant mice on a 129 background (N6 129S1/SvImJ) live a relatively normal lifespan with $100 \%$ surviving $>1$ year (Fig. 3a). This contrasts strongly with the $\sim 50 \%$ of mutants on the B6 background that die by $25-40$ weeks (Fig. 3a). Paralleling the strain-dependent nature of the adult lethality, Gnaol G184S het mice on the 129 background also showed no difference in susceptibility to PTZ kindling compared to wild-type littermates (Fig. 3b). This suggests that one or more modifier loci that differ between B6 and 129 mice affects the penetrance of the lethality and seizure susceptibility phenotypes of the Gnaol G184S mutants. To determine whether the protective or susceptible alleles were dominant, we tested B6/129 F1 mice. No deaths were observed up to 47 weeks in F1 mice regardless of their gender ( $n=16$ per genotype Fig. 3c). Thus, 129 alleles appear to provide a dominant protective effect against the spontaneous death phenotype of the Gnaol ${ }^{+/ G 184 S}$ mutation or, conversely, the C57BL/6J background effect is recessive. Similarly, Gnaol mutant mice on the B6/129 F1 background are protected against PTZ kindling (Fig. 3d, Gehan-Breslow-Wilcoxon $\operatorname{df}(1)=7.9, p<0.01)$. Thus, a single copy of 129 alleles throughout the genome is sufficient for protection in kindling as well as for spontaneous lethality.

\section{Mapping modifier loci}

Evaluating F2 offspring from intercrosses of B6/129 F1 mice carrying the Gnaol G184S mutant allele showed that only 4 out of 60 heterozygous mutant offspring died before one year of age. Genome-wide SNP analysis was done but the small number of mice with the lethal phenotype was insufficient to obtain statistically significant LOD scores (data not shown). Given that $25 \%$ of the F2 mice should be homozygous for B6 alleles at any given locus, 15 mice should have died if there had been a single high-penetrance modifier region; this suggests that there may be more than one modifier.

As an alternative approach to define genetic loci underlying the strain differences, we did a preliminary genome scan on 18 progeny of a single N6 male Gnaol G184S mutation carrier. This analysis was based on the hypothesis that protective 129 alleles should be enriched in the long-lived group. Out of the 11 male and 7 female N7 B6 Gnaol $^{+/ \mathrm{G} 184 \mathrm{~S}}$ mice from Fig. 3a, six were short-lived (i.e., died prior to the survival curve leveling off at $\sim 25$ weeks of age) and 12 were long-lived (i.e., alive past 25-week cutoff). The distribution of 129 (heterozygous) alleles across the genome was assessed by Illumina genotyping (see Supplemental Methods with confirmation markers defined in Table S1). The percent of short-lived and long-lived mice carrying 129 alleles at the indicated chromosomal location is shown in Fig. 4a, b. As expected, all mice retained 129 alleles on $\mathrm{Chr} 8$ (i.e., those linked to the Gnaol locus). The only region that preferentially retained 129 alleles in the long-lived cohort was on $\mathrm{Chr} 17$ at a point after $39 \mathrm{Mb}$ through the end of the chromosome (Fig. 4a, b). Details of other retained regions on Chr 5 and Chr16 are presented in Fig. S3a, b, respectively, and Table S2. This initial analysis was not sufficiently powered to 
Fig. 3 Adult lethality and kindling sensitivity are suppressed on a 129 background. a While B6 (N7) Gnao1 $1^{+/ G 184 S}$ mutant mice die prematurely, mutants on a 129SvImJ strain background do not. b Similarly, the enhanced kindling sensitivity of Gnao $1^{+/ G 184 S}$ mutants is not seen on a 129 strain background. $\mathbf{c}$ The effect of the 129 background is dominant as 129/B6 F1 mice carrying the Gnao1 mutation do not show enhanced adult lethality. d Similarly, the increased kindling sensitivity of Gnao1 $^{+/ G 184 S}$ mutants on the B6 background is partially ameliorated on the F1 background
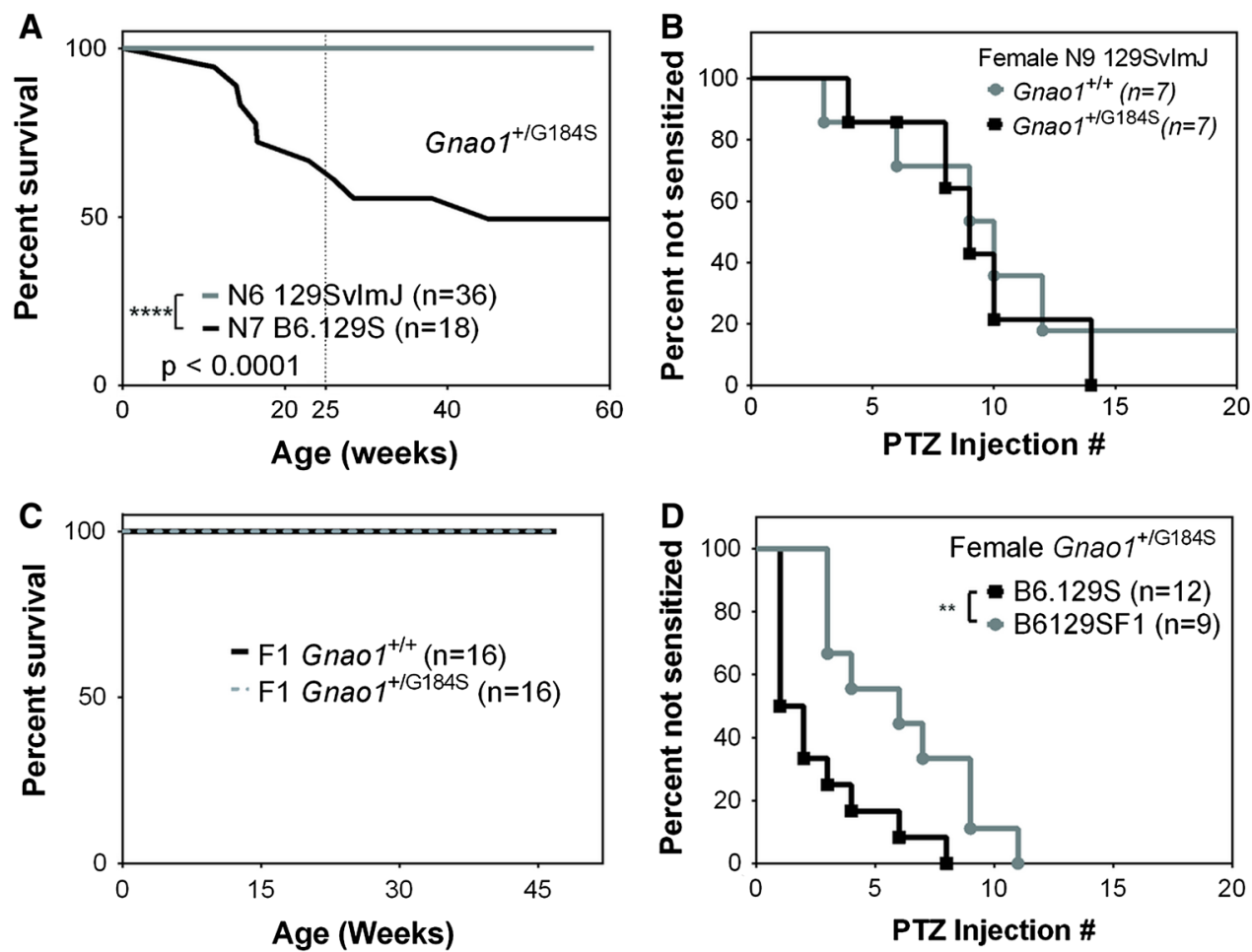

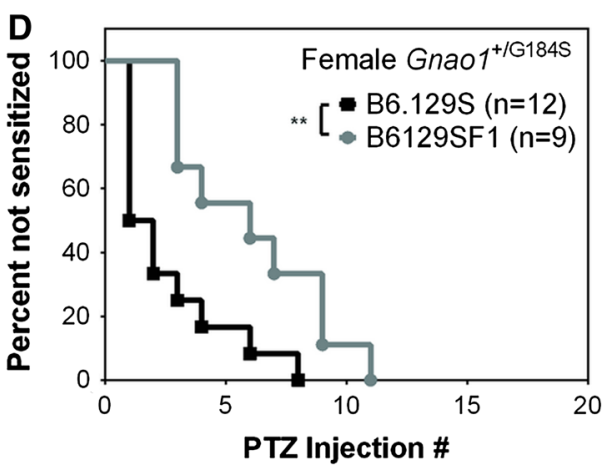

statistically validate the modifier due to the corrections needed for multiple comparisons.

To both confirm and reduce the size of the potential modifier region, we obtained recombinant mice that maintained 129 alleles at 47 and $62 \mathrm{Mb}$ on $\mathrm{Chr} 17$ but were homozygous for B6 alleles at 41 and $70 \mathrm{Mb}$ allowing us to reduce the size of the putative modifier region by half (see details of markers used in Table S1). In an independent experiment with 44 mice, we tested the ability of the (Chr17: 41-70 Mb) subregion to afford protection. This approach of identification followed by validation is supported by the previous literature (Williams and Haines 2011). The presence of 129 alleles in the Chr17: 41-70 Mb subregion protected Gnaol G184S het mice from spontaneous death in the independent cohort (Fig. 4c GehanBreslow-Wilcoxon $\operatorname{df}(1)=4.9, p<0.05)$. The other retained 129 regions (e.g., Chr5 and Chr16 in Fig. 4a) did not influence viability within this cohort (data not shown).

Chr 17 locus also modifies PTZ susceptibility

We next tested the hypothesis that the Chr17 spontaneous death modifier protected against enhanced PTZ susceptibility. In this case, experiments were restricted to females due to their enhanced rate of kindling. The presence of 129 alleles within the $\mathrm{Chr} 17$ : 41-70 Mb modifier region also protected B6 Gnaol mutant hets from kindling (Fig. 5a, Gehan-Breslow-Wilcoxon, $\operatorname{df}(1)=4.28 p<0.05)$. To determine whether the effect of this Chr17: 41-70 Mb locus to protect against kindling depended on the presence of the Gnaol G184S mutant allele, we assessed its effect in Gnaol WT mice. As expected, mice without the Gnaol mutation showed markedly delayed kindling (half-max at injection 7, Fig. 5b vs. than 1 or 2 for the Gnaol mutants, Fig. 5a). There may be a small effect of 129 alleles in the Chr17: 41-70 Mb region on kindling of Gnaol WT mice but there was no statistically significant protection. The striking effect of this region in the presence of the Gnaol mutant prompted us to name this Chr17 locus Mogsl (modifier of $\mathrm{G}$ protein-induced PTZ susceptibility 1) in parallel with the Moel and Moe2 loci which have been previously described for protection against spontaneous seizures induced by sodium channel mutants (Bergren et al. 2005, 2009).

\section{Discussion}

In this report, we show that mice heterozygous for the Gnaol G184S gain-of-function allele have partial perinatal lethality, spontaneous adult lethality, enhanced frequency of epileptiform discharges, and marked sensitization to PTZ kindling. These results are quite interesting in light of the recent finding by Nakamura et al. (2013) that four infants with epileptic encephalopathy (Ohtahara syndrome, EIEE17) have de novo, non-synonymous polymorphisms in GNAO1. Our results with the gain-of-function allele contrast with those for heterozygous Gnaol \pm knockout 

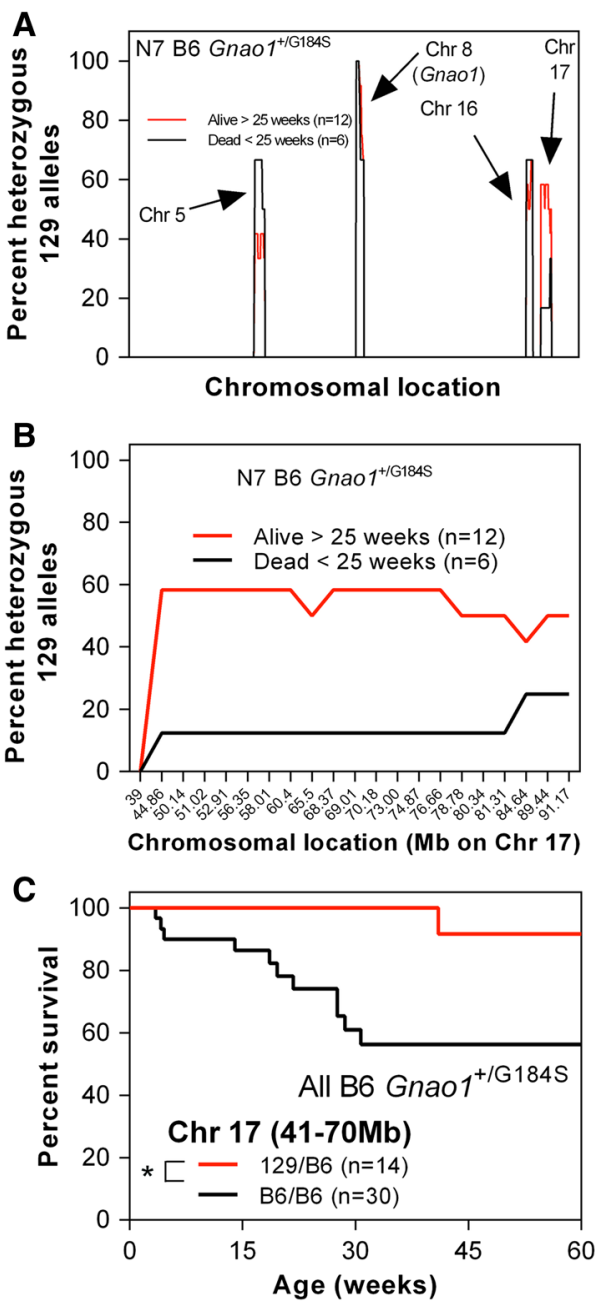

Fig. 4 Mapping a modifier of the strain-dependent lethality on Chromosome 17. A cohort of 18 Gnaol $^{+/ \mathrm{G} 184 \mathrm{~S}}$ mutant mice derived from a single N6 male founder was monitored for longevity. Whole genome SNP analysis was done comparing short-lived (black, dead by 25 weeks) versus long-lived ( $r e d$, alive at 25 weeks). Regions of retained 129S alleles were assessed. a Plots show the percentage of the mutant mice carrying 129 alleles at a particular location in the genome (see $\mathbf{b}$ and Fig. S3 for specific marker locations). All mutant mice had 129 alleles linked to the Gnaol locus on Chr8. b Only one region, Chr 17, showed preferential retention of 129 alleles in the long-lived population. c Confirmation of the association of retained 129 alleles on Chr17 with longer survival was undertaken in an independent cohort of 44 mice. Those mice retaining 129 alleles on Chr 17 at both 47 and $62 \mathrm{Mb}$ (but not at 41 and $70 \mathrm{Mb}$ ) showed increased survival (c Gehan-Breslow-Wilcoxon $\operatorname{df}(1)=4.9$, $p<0.05)$

mice which do not have increased kindling sensitivity and have not been reported to have seizures, though homozygous knockouts do (Valenzuela et al. 1997).

It is of great interest to understand whether the mechanisms of the seizure phenotype in humans with GNAO1 mutations and in our mice $\left(\right.$ Gnaol $\left.^{+/ \mathrm{G} 184 \mathrm{~S}}\right)$ are similar. Our mutant allele is well documented as having enhanced
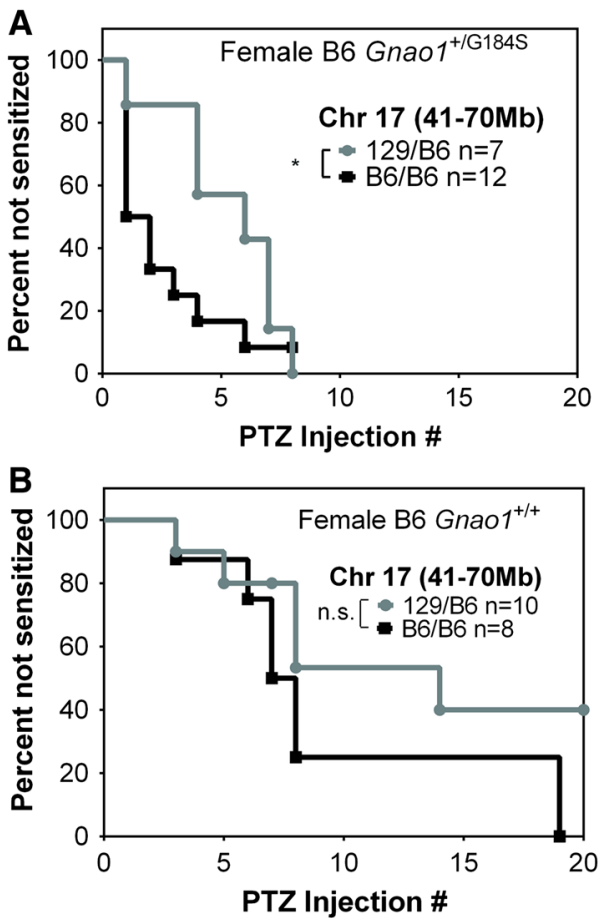

Fig. 5 Chromosome 17 modifier region also affects sensitization to kindling of B6 Gnaol ${ }^{+/ \mathrm{G} 184 \mathrm{~S}}$ mutants but not that of WT mice. a Female Gnaol mutant mice homozygous for B6 alleles at Chr17 (47-62 MB) sensitize to kindling faster than those heterozygous for 129 alleles at those loci. So the $\mathrm{Chr} 17$ region that protects against early lethality also delays sensitization to PTZ kindling (GehanBreslow-Wilcoxon $\operatorname{df}(1)=7.9, p<0.01)$. b The modifier is dependent on $\mathrm{G}_{\mathrm{o}}$ mutation status. b In a sample of similar size to that in $5 \mathrm{~A}$, the Chr 17 modifier region did not significantly alter sensitization to PTZ kindling in B6 Gnaol wt mice

function. In vitro studies with opioid receptors to inhibit both cAMP levels (Clark et al. 2003) and N-type $\mathrm{Ca}^{++}$ channels (Ikeda and Jeong 2004) show profound increases in $\mathrm{G} \alpha_{\mathrm{o}}$ function with the G184S mutation. In brain slices, the mutation also enhances presynaptic inhibition by adenosine (Chen and Lambert 2000) and epinephrine (Goldenstein et al. 2009). In vivo opioid signaling is also enhanced in Gnaol $^{+/ \mathrm{G} 184 \mathrm{~S}}$ mutant mice and reduced in Gnaol $^{+-}$mice (Lamberts et al. 2013). The human GNAO1 mutants alleles analyzed in vitro by Nakamura et al. (2013) were interpreted as providing evidence for loss of function. It is somewhat surprising to see haploinsufficiency in this context since heterozygous Gnaol knockout mice do not show seizures. Indeed, at least two of the four human mutants (N174G and T191-F197del) produced spontaneously lower $\mathrm{Ca}^{++}$channel currents which might instead represent a gain of function. Interestingly, loss-offunction mutations in $\mathrm{P} / \mathrm{Q}-$ type $\mathrm{Ca}^{++}$channels (Cav2.1, CACNA1A) cause absence of seizures in humans; mouse knockouts produce seizures and early lethality (Khosravani and Zamponi 2006). Since $\mathrm{G} \alpha_{\mathrm{o}}$ signaling can suppress both 
$\mathrm{N}$ - and P/Q-type calcium channel activity, a gain-of-function allele in GNAO1 is potentially a pro-seizure mechanism.

While brain morphology changes are seen in 3 of the 4 cases of EIEE17, we did not see any gross alterations in brain morphology in our Gnaol mutant mice nor were such changes seen in Gnaol homozygous knockouts (Valenzuela et al. 1997). It is not clear whether the effect of the Gnaol G184S mutation is through alterations in brain development or simply through enhanced function. Increased Gnaol signaling to $\mathrm{N}-$ and P/Q-type $\mathrm{Ca}^{++}$ channels would be expected to suppress neurotransmitter release which might be expected to have antiepileptic actions. However, both gain and loss of sodium channel activity can lead to seizures (Meisler and Kearney 2005), so a similar phenomenon may be at play here. If enhanced $\mathrm{G} \alpha_{\mathrm{o}}$-mediated presynaptic inhibition occurred in inhibitory (GABAergic) neurons, as has been proposed for the epileptogenic Nav1.1 LOF mutants (Yu et al. 2006), one could see hyper-excitability due to suppressed inhibitory tone. This is consistent with our prior observation of reduced in vitro excitability in hippocampal slices from mice carrying our mutation (Goldenstein et al. 2009). In that model, the ability of epinephrine to suppress hippocampal epileptiform discharges was enhanced in the $\mathrm{Ga}_{\mathrm{o}}^{+/ \mathrm{G} 184 \mathrm{~S}}$ mutants. Since inhibitory signaling was abrogated in that model with picrotoxin, the pro-seizure behavior that we see here would have been missed if the mechanism is suppression of inhibitory signaling. Further study will be necessary to distinguish between structural and functional mechanisms.

The present study also suggests a role for $\mathrm{G}_{\mathrm{o}}$ signaling in sudden unexplained death in epilepsy (SUDEP). One proposed mechanism of SUDEP is post-ictal bradycardia or hypoventilation (Surges and Sander 2012). In addition to the increase in interictal epileptiform discharges and spontaneous death reported here, the $\mathrm{G} \alpha_{\mathrm{o}}$ G184S mutation also enhances bradycardia to muscarinic and adenosine signaling (Fu et al. 2006). Increased bradycardia or effects on brainstem control of ventilation could contribute to the lethality that we observe. Clearly, further study of this is needed.

In addition to the effect of the Gnaol G184S mutation, we have identified a second locus that ameliorates the phenotype in our Gnaol mutant mice. Both spontaneous deaths and enhanced kindling sensitization were substantially mitigated in the presence of 129 alleles derived from a $29 \mathrm{Mb}$ modifier region on $\mathrm{Chr} 17$, which we term modifier of $\mathrm{G}$ protein-induced seizures $1(\operatorname{Mogs} 1)$. It is of interest that the protective modifier alleles on $\mathrm{Chr} 17$ come from the 129 strain which is more sensitive to PTZ kindling compared to C57Bl/6J. This, however, is relatively common in epilepsy models (Bergren et al. 2009). The modifier region appears to have its effect on PTZ susceptibility primarily in Gnaol mutant mice. Also, Mogs 1 seems not to influence effects of acute PTZ administration as it was not found in a genomic analysis of acute PTZ-induced seizures (high dose- $80 \mathrm{mg} / \mathrm{kg}$ ) in B6 vs DBA mice (Ferraro et al. 1999). These observations suggest that this modifier region (Mogsl) may have a unique interaction with $\mathrm{G}_{\mathrm{o}}$ function or perhaps in PTZ kindling-type remodeling mechanisms but not in acute seizures. Defining the specific genes and alleles that underlie its effect will be important.

The Mogs1 region (Chr17: 41-70 Mb) includes the coding sequences of $\sim 130$ known proteins. One that was an obvious candidate was Rab5a, the gene product of which has been proposed as a novel $G_{o}$ effector in Drosophila (Purvanov et al. 2010). Loss of an effector molecule could easily suppress a gain-of-function mutation in $\mathrm{G}_{\mathrm{o}}$. There are no non-synonymous coding SNPs in Rab5a between 129 and B6 strains. Despite several SNPs in the $5^{\prime}$ and $3^{\prime}$ UTR, there were no changes in mRNA expression as detected by quantitative real-time PCR (data not shown). Thus, Rab5a does not appear to be the modifier gene. To develop a list of other possible candidates, we looked for non-synonymous SNPs or splice site variants identified in the Sanger informatics website. Three proteins have altered stop codons. Two genes, 9130008F23Rik K55* and Trem2 149*, have premature stops, while Yipf3 lost a stop codon, * 202 W. Polh has a variant in a splice site, while Ttbkl, Tbcld5, and Lrfn2 have in-frame dels in the $129 \mathrm{~S}$ sequence. For non-synonymous SNPs, the predicted effect on protein function was assessed using Provean (Choi et al. 2012). Seven genes Pgk2, Gpr115, Gm5093, BC048355, $\operatorname{Lrfn} 2, K c n h 8$, and $V m n 2 r 118$ were predicted to have nonsynonymous SNPs disruptive of protein function in the 129S strain. Gprl15 is an obvious high-priority candidate. In addition to documented SNPs between 129S and B6 strains, we also generated a candidate list of 20 genes based on known CNS function or roles in GPCR signaling. These lists represent a starting point for future candidate analysis in conjunction with higher resolution experimental mapping of Mogsl. Whole exome and targeted exome sequencing is currently underway.

In summary, we demonstrate that a Gnaol gain-offunction mutation causes a sudden death phenotype with associated seizures, increases sensitivity to PTZ kindling, and enhanced frequency of spontaneous epileptiform discharges in C57BL/6J mice. This provides a novel experimental model with potential relevance to a subset of Ohtahara syndrome (EIEE17) and possibly SUDEP in humans. Further study of the mechanism of this mouse model, as well as identification of the genes involved in the modifier locus on Chr17, should provide insights into the complex genetic basis of human epilepsy. 
Acknowledgments This work was supported by the National Institutes of Health (R01 GM036592-25 to R. R. N.), the PhRMA Foundation (predoctoral award to J. H. K.), and National Institutes of Health through the University of Michigan's Cancer Center Support Grant (5 P30 CA46592) by the use of the DNA sequencing core. The authors have no conflicts of interest related to this work. We would also like to thank Drs. Bob Lyons and Susan Dagenais (University of Michigan DNA Sequencing core) for help in design and analysis of genome-wide SNP data, Dr. David Burke (University of Michigan) for thoughtful input on the design of the PCR SNP testing and F2 analysis, and Dr. Tom Gettys (Medical University of South Carolina) for the $\mathrm{G} \alpha_{\mathrm{o}}$ antibody. Drs. Miriam Meisler and Lori Isom (University of Michigan) provided helpful comments on the manuscript.

\section{References}

Bergren SK, Chen S, Galecki A, Kearney JA (2005) Genetic modifiers affecting severity of epilepsy caused by mutation of sodium channel Scn2a. Mamm Genome 16:683-690

Bergren SK, Rutter ED, Kearney JA (2009) Fine mapping of an epilepsy modifier gene on mouse Chromosome 19. Mamm Genome 20:359-366

Bialer M, White HS (2010) Key factors in the discovery and development of new antiepileptic drugs. Nat Rev Drug Discov 9:68-82

Birnbaumer L (2007) Expansion of signal transduction by G proteins. The second 15 years or so: from 3 to 16 alpha subunits plus betagamma dimers. Biochim Biophys Acta 1768:772-793

Chen H, Lambert NA (2000) Endogenous regulators of G protein signaling proteins regulate presynaptic inhibition at rat hippocampal synapses. Proc Natl Acad Sci USA 97:12810-12815

Chen Y, Liu Y, Cottingham C, McMahon L, Jiao K, Greengard P, Wang Q (2012) Neurabin scaffolding of adenosine receptor and RGS4 regulates anti-seizure effect of endogenous adenosine. J Neurosci 32:2683-2695

Clark MJ, Harrison C, Zhong H, Neubig RR, Traynor JR (2003) Endogenous RGS protein action modulates mu-opioid signaling through Galphao. Effects on adenylyl cyclase, extracellular signal-regulated kinases, and intracellular calcium pathways. J Biol Chem 278:9418-9425

Dhir A (2012) Pentylenetetrazol (PTZ) kindling model of epilepsy. Current protocols in neuroscience/editorial board, JN Crawley et al. Chapt. 9, Unit 9, 37

Engel J Jr (1996) Surgery for seizures. N Engl J Med 334:647-652

Ferraro TN, Golden GT, Smith GG, St Jean P, Schork NJ, Mulholland N, Ballas C, Schill J, Buono RJ, Berrettini WH (1999) Mapping loci for pentylenetetrazol-induced seizure susceptibility in mice. J Neurosci 19:6733-6739

Fu Y, Huang X, Zhong H, Mortensen RM, D'Alecy LG, Neubig RR (2006) Endogenous RGS proteins and Galpha subtypes differentially control muscarinic and adenosine-mediated chronotropic effects. Circ Res 98:659-666

Golarai G, Cavazos JE, Sutula TP (1992) Activation of the dentate gyrus by pentylenetetrazol evoked seizures induces mossy fiber synaptic reorganization. Brain Res 593:257-264

Goldenstein BL, Nelson BW, Xu K, Luger EJ, Pribula JA, Wald JM, O'Shea LA, Weinshenker D, Charbeneau RA, Huang X et al (2009) Regulator of $G$ protein signaling protein suppression of Galphao protein-mediated alpha2A adrenergic receptor inhibition of mouse hippocampal CA3 epileptiform activity. Mol Pharmacol 75:1222-1230

Grecksch G, Becker A, Schroeder H, Kraus J, Loh H, Hollt V (2004) Accelerated kindling development in mu-opioid receptor deficient mice. Naunyn Schmiedebergs Arch Pharmacol 369:287-293
Hirtz D, Thurman DJ, Gwinn-Hardy K, Mohamed M, Chaudhuri AR, Zalutsky R (2007) How common are the "common" neurologic disorders? Neurology 68:326-337

Ikeda SR, Jeong SW (2004) Use of RGS-insensitive Galpha subunits to study endogenous RGS protein action on G-protein modulation of $\mathrm{N}$-type calcium channels in sympathetic neurons. Methods Enzymol 389:170-189

Khosravani H, Zamponi GW (2006) Voltage-gated calcium channels and idiopathic generalized epilepsies. Physiol Rev 86:941-966

Lamberts JT, Smith CE, Li MH, Ingram SL, Neubig RR, Traynor JR (2013) Differential control of opioid antinociception to thermal stimuli in a knock-in mouse expressing regulator of G-protein signaling-insensitive Galphao protein. J Neurosci 33:4369-4377

Lee CH, Javed D, Althaus AL, Parent JM, Umemori H (2012) Neurogenesis is enhanced and mossy fiber sprouting arises in FGF7-deficient mice during development. Mol Cell Neurosci 51:61-67

Li T, Steinbeck JA, Lusardi T, Koch P, Lan JQ, Wilz A, Segschneider M, Simon RP, Brustle O, Boison D (2007) Suppression of kindling epileptogenesis by adenosine releasing stem cellderived brain implants. Brain 130:1276-1288

Loscher W (2011) Critical review of current animal models of seizures and epilepsy used in the discovery and development of new antiepileptic drugs. Seizure 20:359-368

Machin D, Cheung YB, Parmar MKB, Parmar MKB (2006) Survival analysis: a practical approach, 2nd edn. Chichester, Hoboken

Masino SA, Li T, Theofilas P, Sandau US, Ruskin DN, Fredholm BB, Geiger JD, Aronica E, Boison D (2011) A ketogenic diet suppresses seizures in mice through adenosine $\mathrm{A}(1)$ receptors. J Clin Investig 121:2679-2683

Meisler MH, Kearney JA (2005) Sodium channel mutations in epilepsy and other neurological disorders. J Clin Investig 115:2010-2017

Michelucci R, Pasini E, Riguzzi P, Volpi L, Dazzo E, Nobile C (2012) Genetics of epilepsy and relevance to current practice. Curr Neurol Neurosci Rep 12:445-455

Morimoto K, Fahnestock M, Racine RJ (2004) Kindling and status epilepticus models of epilepsy: rewiring the brain. Prog Neurobiol 73:1-60

Nakamura K, Kodera H, Akita T, Shiina M, Kato M, Hoshino H, Terashima H, Osaka H, Nakamura S, Tohyama J et al (2013) De Novo mutations in GNAO1, encoding a Galphao subunit of heterotrimeric $G$ proteins, cause epileptic encephalopathy. Am J Hum Genet 93:496-505

Ormandy GC, Jope RS (1991) Pertussis toxin potentiates seizures induced by pilocarpine, kainic acid and $N$-methyl-D-aspartate. Brain Res 553:51-57

Peterson SL, Albertson TE (1998) Neuropharmacology methods in epilepsy research. CRC, Boca Raton

Valenzuela D, Han X, Mende U, Fankhauser C, Mashimo H, Huang P, Pfeffer J, Neer EJ, Fishman MC (1997) G alpha(o) is necessary for muscarinic regulation of $\mathrm{Ca} 2+$ channels in mouse heart. Proc Natl Acad Sci USA 94:1727-1732

Wilczynski GM, Konopacki FA, Wilczek E, Lasiecka Z, Gorlewicz A, Michaluk P, Wawrzyniak M, Malinowska M, Okulski P, Kolodziej LR et al (2008) Important role of matrix metalloproteinase 9 in epileptogenesis. J Cell Biol 180:1021-1035

Williams SM, Haines JL (2011) Correcting away the hidden heritability. Ann Hum Genet 75:348-350

Yu FH, Mantegazza M, Westenbroek RE, Robbins CA, Kalume F, Burton KA, Spain WJ, McKnight GS, Scheuer T, Catterall WA (2006) Reduced sodium current in GABAergic interneurons in a mouse model of severe myoclonic epilepsy in infancy. Nat Neurosci 9:1142-1149 